\title{
USO DE FACEBOOK Y LA AUTOPERCEPCIÓN DE LA IMAGEN CORPORAL EN MUJERES
}

\section{Use of facebook and the self perception of body image in women}

\author{
Jazmin del Rosario Infante Faura* \\ Carmen Margarita Ramírez Luna**
}

\begin{abstract}
Resumen
La presente investigación es un estudio cuantitativo de tipo aplicado, transversal y descriptivo correlacional. El objetivo fue determinar la relación entre el uso de Facebook y la autopercepción de la imagen corporal en un grupo de estudiantes universitarias. Para el caso, se aplicó una escala que mide el uso de Facebook y un cuestionario de imagen corporal, a 106 estudiantes cuyas edades comprendían entre 17 y 24 años, del primer, quinto y décimo ciclo de estudios de las carreras de Psicología y Comunicaciones de una Universidad Privada de Lima Metropolitana. Los resultados muestran una relación positiva y significativa entre el uso de Facebook y la autopercepción negativa de la imagen corporal en mujeres $(r=0.247 ; p<0.05)$..
\end{abstract}

Palabras clave: Uso de Facebook, autopercepción de la imagen corporal, estudiantes universitarias.

\begin{abstract}
The present research is a quantitative study and an applied, transversal and descriptive correlational study about the use of Facebook and self - perception of the body image in a group of university students. The purpose was to determine the relationship between the use of Facebook and self-perception of the body image. For this case it was used a scale that measured the used of Facebook and a body shape questionnaire. These were applied to 106 students whose ages ranged from 17 to 24 years old, from the first, fifth and tenth semester of the undergraduate department of Psychology and Communications from a Private University of Lima Metropolitana. The results show a positive and significant relationship between the use of Facebook and negative self-perception of body image in women $(r=0.247 ; p<0.05)$.
\end{abstract}

Keywords: Use of Facebook, self-perception of body image, university students.

* Psicóloga y administradora de empresas.jazmininfantef@gmail.com

** Psicóloga y profesora de educación inicial.carmenramirezluna@hotmail.com

Artículo basado en la tesis de licenciatura sustentada por las autoras. 


\section{INTRODUCCIÓN}

En la actualidad el uso de Facebook influye notablemente en la vida de las personas; sin embargo, muy poca investigación se ha realizado sobre este tema en constante evolución. Dentro de las redes sociales, el Facebook es la más usada a nivel mundial, ya que el $71 \%$ de usuarios de internet, tiene un perfil de Facebook (Duggan \& Smith, 2014). Estos sitios permiten a las mujeres la oportunidad de crear su propio espacio de imágenes, así como ver y criticar el contenido creado por los pares y de los famosos a los que admiran.

Si bien es cierto, existen pocas evidencias sobre la influencia del uso de Facebook, las existentes señalan que su uso podría afectar la autoestima, personalidad, potenciando trastornos de alimentación y la insatisfacción de la imagen corporal. Esto posiblemente se debe a que juega un papel desmesurado en la comunicación de los estereotipos culturales sobre la estética de la imagen corporal. Esto nos motivó a investigar sobre la relación entre el uso de Facebook y la autopercepción de la imagen corporal. Con los hallazgos que se evidencien se podrían crear programas de prevención e intervención, que ayuden a reducir la incidencia en nuestra población.

Con el interés de generar más evidencias al respecto, el objetivo de la presente investigación es determinar la relación entre el uso de la red social Facebook y la autopercepción de la imagen corporal en una población universitaria.

\section{REDES SOCIALES}

\section{Comunicación digital}

Este concepto es crucial debido a que de este se desprende el de redes sociales. En términos generales, la comunicación digital, afirma Cárdenas (2015), se remite al proceso comunicativo que se da a través de un dispositivo y, por tanto, aunque toma como referencia la comunicación personal física, esta comunicación también posee características propias, las que se dan como parte de la adaptación de los usuarios a un espacio digital.

El concepto definido por December (1997), nos dice que la comunicación mediada por computadora es un proceso en el que intervienen personas situadas en contextos particulares y vinculadas en procesos que modifican los medios de comunicación para una variedad de propósitos.

La definición de December se mantiene vigente dado que no excluye a los dispositivos móviles que cada vez son más comunes. Esta definición abarca la comunicación mediada por computadora, sea esta una PC, un celular o una tablet ya que todos estos dispositivos encajan, de manera general, en la definición de computadora.

\section{Uso de Internet}

Antes de hablar del uso de las redes sociales, es importante tener en cuenta el uso de internet tanto a nivel mundial, como regional.

De acuerdo a Internet World Stats (2016) y teniendo en cuenta la población mundial de 7,259,902,243, el $46.4 \%$ de personas en el mundo son usuarios de internet, presentando un incremento del $832.5 \%$ desde el 2000 hasta el 2015. En tal sentido y siendo nuestra población a evaluar perteneciente a la región latinoamericana, se puede observar el gran incremento de uso de internet que alcanza el $1808.4 \%$ en dicha área geográfica.

Tabla 1

Uso de Internet a Nivel Mundial

\begin{tabular}{lcc}
\hline Regiones & Penetración & $\begin{array}{c}\text { Crecimiento } \\
(\mathbf{2 0 0 0 - 2 0 1 5})\end{array}$ \\
\hline África & $28.60 \%$ & $7231.30 \%$ \\
Asia & $40.20 \%$ & $1319.10 \%$ \\
Europa & $73.50 \%$ & $474.90 \%$ \\
Medio Oriente & $52.20 \%$ & $3649.80 \%$ \\
Norteamérica & $87.90 \%$ & $190.40 \%$ \\
Latinoamérica/Caribe & $55.90 \%$ & $1808.40 \%$ \\
Oceanía & $73.20 \%$ & $256.90 \%$ \\
Total Mundial & $46.40 \%$ & $832.50 \%$ \\
\hline
\end{tabular}

Fuente: Internet World Stats, 2016

De acuerdo a la misma investigación, en nuestro país, de los poco más de 30 millones de habitantes, 16 millones son usuarios de internet representando esto el 
$52.6 \%$ de los cuales el mismo porcentaje hacen uso de la red social Facebook (Internet World Stats, 2016).

\section{Redes Sociales}

Morduchowicz (2010) señala que en 1995 apareció la primera red social denominada Classmates. com y fue creada por un ex estudiante universitario para comunicarse con sus compañeros de clase. Posteriormente en 1997, se creó Sixdegrees.com, primer sitio de redes sociales con perfiles y lista de amigos. El 2001 y el 2002 aparecen los primeros sitios web basados en círculos de amigos en línea. Estos sitios se popularizaron el 2003 con la llegada de redes sociales específicas que ofrecen espacios de intereses afines. Hasta el 2010 existían 200 redes sociales en el mundo con más de 800 millones de usuarios.

De acuerdo con Boyd \& Ellison (2007), las redes sociales son servicios web que permiten a los individuos construir un perfil público o semipúblico dentro de un sistema, articular una lista de usuarios con quienes ellos pueden compartir una conexión, ver y navegar en su lista de enlaces y en aquellas hechas por otros dentro del sistema. La naturaleza y la nomenclatura de estas conexiones pueden variar según el sitio.

En estas redes sociales, a fin de interactuar unos con otros, los usuarios deben crear perfiles que son alimentados con información que voluntariamente las personas van almacenando en ellos. Estos perfiles se convierten en la representación del usuario, y a partir de ellos se va construyendo su imagen en la red (Cárdenas, 2015).

En estas plataformas digitales se comprueba de forma patente la acción comunicativa de la que habla Costa (2009), ya que las acciones de los usuarios, que son reflejadas y almacenadas en los perfiles de cada uno, son las que van conformando un historial que comunica y genera la imagen de los usuarios que tienen los individuos que conforman su entorno. Asimismo, a diferencia de las relaciones personales en el mundo offline, dentro de las redes sociales resulta accesible en cualquier momento buscar, visualizar e incluso estudiar el comportamiento de los individuos.

Como señalan Acquisti \& Gross (2006), una red social es una comunidad de internet donde los individuos interactúan, usualmente a través de perfiles que representan su persona pública (y su red de contacto) con otros. A pesar que el concepto de comunidades basadas por computadora data de los primeros días de las redes informáticas, solo luego de la llegada del internet comercial esas comunidades conocieron el éxito público.

El entorno de estas redes sociales está marcado por la dinamicidad, ya que los usuarios no solo pueden buscar información de otros en cualquier momento, sino que el proceso comunicativo que establecen con otros usuarios se da en tiempo real; este necesariamente no es de uno a uno, sino que muchas veces también se da de uno a varios y viceversa (Cárdenas, 2015).

Entorno a esto, Ressler \& Glazer (2010) sostienen que las redes sociales pueden ser definidas como una constelación de herramientas basadas en Internet, que ayudan a los usuarios a conectar, colaborar y comunicarse con otros en tiempo real. Las redes sociales le permiten a uno participar en un diálogo o conversación digital activa; en contraste con el monólogo digital pasivo, por ejemplo, el leer una página web estática.

Otro de los cambios que se observan en el proceso comunicativo de las redes sociales son los actores, quienes, tal y como se da en la comunicación bilateral, pueden ser emisores y receptores casi al mismo tiempo. De forma similar, en las redes sociales, a diferencia de las plataformas virtuales primigenias, los usuarios son, al mismo tiempo, productores y consumidores. Ejemplo de esto es que los individuos para ser miembros de estas redes sociales, deben brindar información para crear sus perfiles, y de esa forma ya están creando contenido que es valioso y parte clave para el funcionamiento de la propia red social (Cárdenas, 2015).

Según datos estadísticos recogidos por Duggan \& Smith (2014), al 2013 Facebook sigue siendo la red social más usada seguida por Linkedin, Pinterest, Twitter e Instagram. 
Figura 1. Redes sociales 2012-2013, Porcentaje de adultos que usan redes sociales por año.

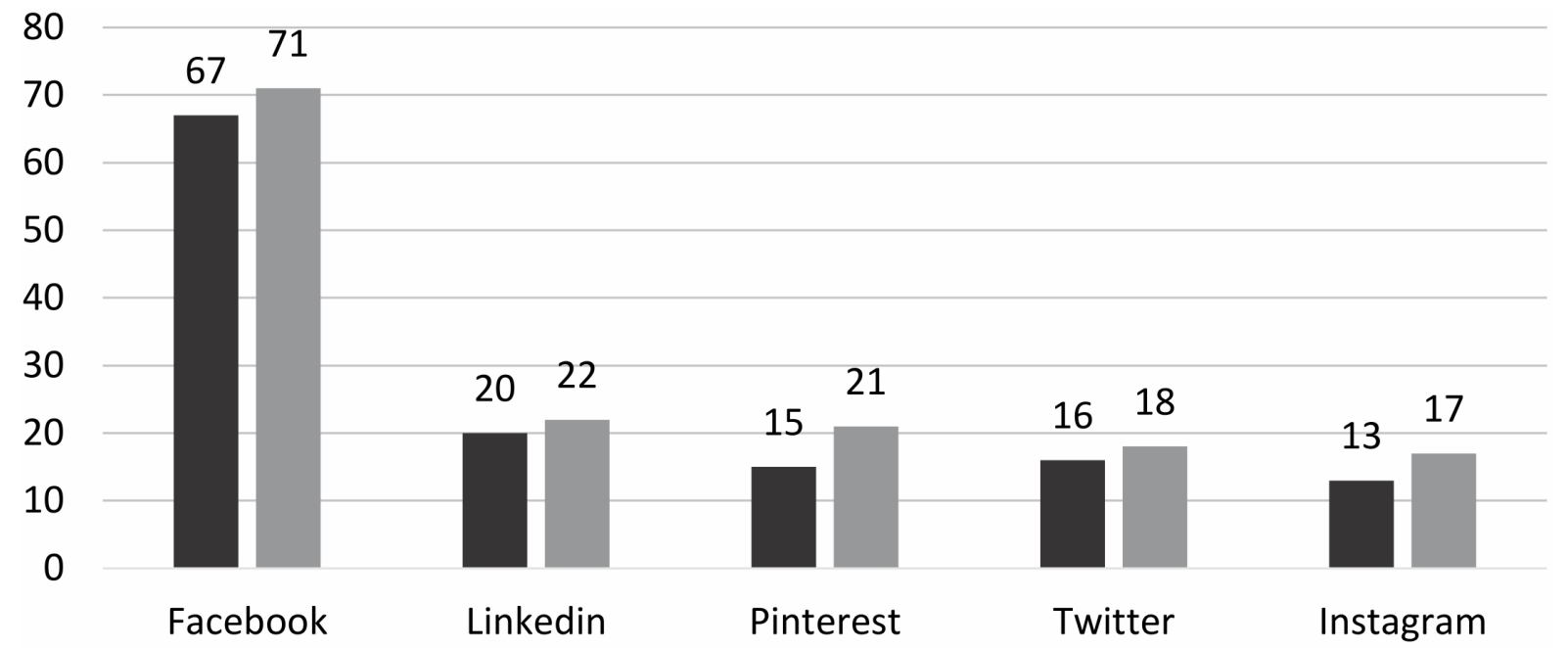

$2012 \square 2013$

Adicionalmente, en el mismo estudio, Duggan $\&$ Smith (2014), establecen que Facebook es usado de manera muy similar tanto en hombres como en mujeres. Dentro de las redes sociales que se muestran en la Figura 1, los usuarios de Facebook e Instagram son los que más hacen uso de ellas. Por ejemplo, el $63 \%$ de los usuarios de Facebook visitan el sitio web por lo menos una vez al día y el $40 \%$ lo visita reiteradas veces durante el día. Otro dato interesante está relacionado con la cantidad de redes sociales que usa una misma persona. En tal sentido, el $42 \%$ de los adultos usuarios de internet usan 2 o más de estas redes sociales. Dentro de los que usan solo una red social, el $82 \%$ es usuario de Facebook.

\section{Facebook}

Esta es una red social creada en 2004 por Mark Zuckerberg, Dustin Moskovitz y Chris Hughes, mientras ellos eran estudiantes en la Universidad de Harvard. En un inicio tuvo como fin, brindarles a los alumnos de esa universidad un lugar donde ellos pudieran mantenerse en contacto con sus compañeros y en el que pudieran compartir información vinculada a los cursos (Orellana, 2012).

Al principio, esta red social estaba solo disponible como medio de comunicación entre los estudiantes de esa universidad (Akyildiz \& Argan, 2012). No obstante, pronto Facebook estuvo disponible para estudiantes de otras universidades y en 2005, pudo ser utilizada por alumnos de educación secundaria (Hebrang, 2013). En setiembre de 2006, el sitio fue abierto al público a nivel global (personas mayores de 13 años); aunque esta red social no llega a países como China, en donde el sitio está bloqueado (Delaney, 2013).

A pesar que la apariencia de esta red social ha variado con el tiempo, lo que se mantiene es que los usuarios se inscriben gratuitamente y reciben una página personal, llamada perfil. Cada individuo decide cuánta información personal añade a su cuenta de Facebook. Sin embargo, existen datos esenciales para la inscripción en la red social como son correo electrónico,nombre, edad y sexo(Delaney,2013).Entre la información adicional se encuentra la orientación sexual, religiosa, política; asimismo, se puede agregar una foto que identifique a la persona. El usuario elige acerca de la privacidad de la data brindada a la red social (es decir, si la pueden ver el público en general, solo sus amigos, o únicamente la persona misma).

Luego que los miembros se han registrado, pueden empezar a recibir invitaciones de amistad, lo que contribuye a que el usuario amplíe su lista de contactos. También los individuos pueden publicar en sus páginas de perfil: texto, enlaces de páginas webs, 
fotos o una combinación de todos estos. Facebook prácticamente no tiene límites en torno a la cantidad de data (como fotos o videos) que los usuarios pueden colgar (Cárdenas, 2015).

Esta red social, en tiempo real, reúne todas estas publicaciones hechas por los usuarios, las que se visualizan en la sección "noticias". No solo se despliega el contenido subido por los usuarios, sino que también las publicaciones de las organizaciones que los usuarios siguen en esta red (DiVall \& Kirwin, 2012). Esto último se consigue luego de que los miembros dan clic en el botón de "me gusta"; con lo cual, acuerdan seguir los contenidos de las páginas de Facebook de tales organizaciones.

Según Bartholomew \& otros (2012), existen siete usos primarios que tiene esta red social:

a) El primero se centra en la posibilidad que tienen los usuarios de establecer y mantener lazos sociales con otros miembros.

b) Asimismo, Facebook permite que los individuos encuentren temas que les son afines en grupos o eventos formados al interior de esta red social, e interactuar con usuarios de gustos similares.

c) Otro de los usos es mirar y publicar fotos y videos.

d) Los miembros también usan Facebook para conectarse con otras aplicaciones, juegos y cuestionarios. e) De igual forma, las personas emplean esta red social para saber más acerca de gente que conoció en el mundo offline.

f) Además, se pueden establecer relaciones con alguna persona, por medio de los contactos en común (dentro de la red) que se puedan tener con ella.

g) Finalmente, los miembros pueden compartir y revisar las publicaciones que hacen sus contactos

Otros estudios realizados en torno a la red social, señalan que Facebook es atractivo a los usuarios porque genera: curiosidad entre ellos por ver las fotos que sus contactos comparten; placer de poder comunicarse con otros usuarios y emoción debido a que pueden enviar regalos y jugar con otros (Palmer \& Koening-Lewis, 2009).

Asimismo, una investigación indica que los individuos miembros de Facebook pasan en tal red social un promedio 32 minutos al día, y la mayoría de ellos se conecta a ella al menos una vez por día (Park \& Cho, 2012).

A continuación, se presenta un gráfico donde se observa el crecimiento del uso de esta red social a nivel mundial desde el primer trimestre del 2013 al primer trimestre del 2015, representado en millones de usuarios activos. (Internet World Stats, 2016):

Figura 2. Millones de usuarios activos de Facebook. Años 2013 - 2015

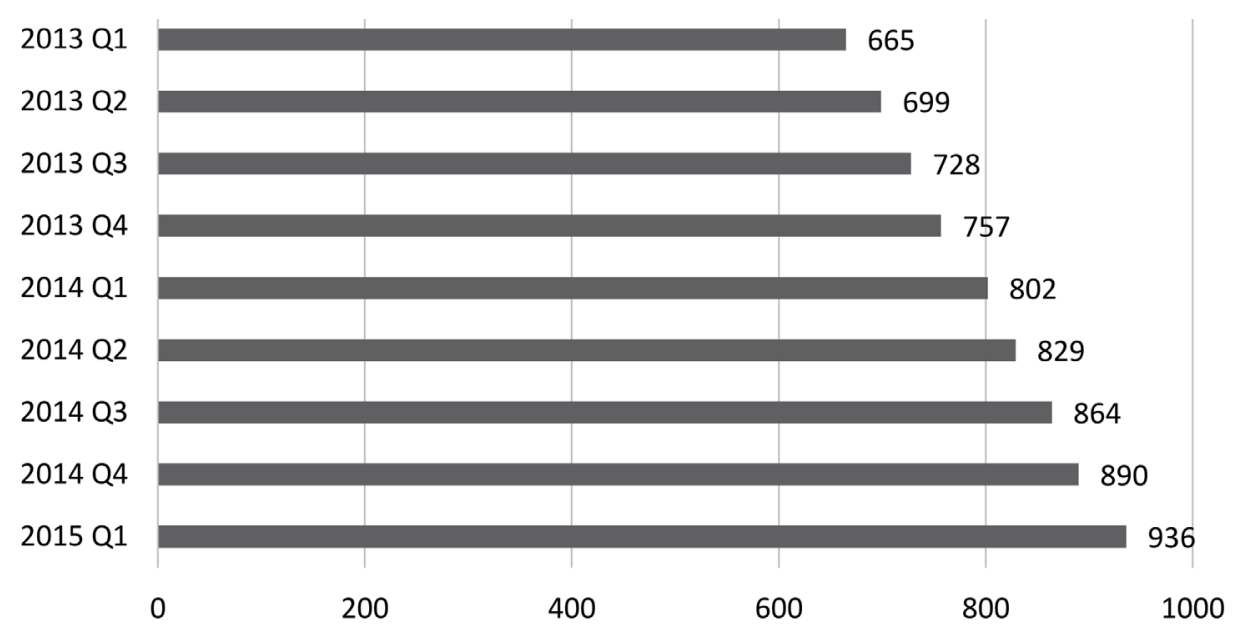




\section{IMAGEN CORPORAL}

\section{Definición de imagen corporal}

La imagen corporal es la representación mental que cada individuo tiene de su propio cuerpo.

La definición más utilizada fue formulada por el neurólogo alemán Paul Schilder en 1950 donde postula que la imagen del cuerpo humano es la foto de nuestro propio cuerpo que nos formamos en la mente, esto quiere decir que es la manera en que nos vemos a nosotros mismos (Escobar, 2008).

Por lo tanto, la imagen corporal es un constructo psicológico complejo, que se refiere a cómo la autopercepción del cuerpo/apariencia genera una representación mental, compuesta por un esquema corporal perceptivo y así como las emociones, pensamientos y conductas asociadas.

\section{Componentes de la imagen corporal}

La imagen corporal es la representación mental que cada individuo tiene de su propio cuerpo, e incluye tres componentes: perceptual, cognitivo y conductual; según Baile (2003):

a) El componente perceptual (aspecto físico), hace referencia a la precisión con que se percibe el tamaño corporal de diferentes partes corporales o del cuerpo en su totalidad.

b) El componente cognitivo (aspecto psicológico), incluye sentimientos, cogniciones y valoraciones que provoca el cuerpo, principalmente el tamaño corporal, el peso, partes del cuerpo o cualquier otro aspecto de la apariencia física (por ejemplo, satisfacción, preocupación, ansiedad, etc.).

c) El componente conductual (aspecto social), se refiere a las conductas originadas por la percepción del cuerpo y los sentimientos asociados (por ejemplo, conductas de evitación de situaciones en las que se tenga que exponer el cuerpo, compararse continuamente con los demás, vestir ropa ancha para no marcar la silueta, pesarse compulsivamente, etc.)

Integrando los tres componentes, se concluye que la imagen corporal hace referencia a la percepción que tenemos de todo el cuerpo y de cada una de sus partes, como del movimiento y límites de éste, a la experiencia subjetiva de actitudes, pensamientos, sentimientos $\mathrm{y}$ valoraciones que hacemos y sentimos, y al modo de comportarnos derivado de las cogniciones y los sentimientos que experimentamos.

Factores que influyen en la formación de la imagen corporal

Quiles (2003), afirma que existen factores internos y externos:

\section{Factores internos}

La formación de la imagen corporal es un proceso dinámico, en el que el cambio de uno de los factores que la componen altera los demás.

El resultado final dependerá de la interacción de los siguientes factores:

a) El propio cuerpo: Se refiere a todos los datos objetivos posibles con los que se puede describir un cuerpo, así como al aspecto funcional de los mismos.

b) La percepción del cuerpo: En la formación de la imagen corporal tiene especial relevancia los aspectos a los que se presta una especial atención y los que se omiten. De modo que seleccionando los aspectos positivos del cuerpo probablemente se creará una imagen positiva y, por el contrario, si tan sólo se perciben los aspectos negativos la imagen no será satisfactoria.

c) Pensamientos sobre el cuerpo: Hace referencia a las creencias e ideas sobre el propio cuerpo. Se puede limitar a los datos objetivos ("Tengo estrías en los muslos"), o a juicios de valor ("Como no estoy delgado, nadie me va a querer").

d) Emociones en relación con el cuerpo: Si la percepción y los pensamientos que se tienen sobre uno mismo son erróneos puede dar lugar a emociones perturbadoras.

e) Comportamientos con relación al cuerpo: si la percepción, los pensamientos y el estado de ánimo respecto al cuerpo son incorrectos, probablemente los comportamientos también 
lo serán. Las personas muy preocupadas por su figura evitan situaciones e intentan ocultar su cuerpo. Esta evitación refuerza la imagen negativa que se tiene de uno mismo.

\section{Factores externos}

La moda, los medios de comunicación, la publicidad y muchos otros factores socioculturales contribuyen a que la delgadez corporal se asocie a prestigio social y se impone a través de:

a) Los medios de comunicación social; que hacen posible que el mensaje de la delgadez como ideal de belleza llegue a millones de mujeres y hombres a través del cine, la televisión, fotografías, etc.

b) La moda del vestir; presentada por modelos de dimensiones corporales reducidas, casi esqueléticas y la dificultad de encontrar tallas más grandes como si el cuerpo tuviera que adaptarse a la moda.

c) La transmisión oral de dietas y el gran número de horas que dedicamos a hablar de la gordura o de la delgadez en los distintos grupos a los que pertenecemos: familias, amigos, compañeros.

d) La exigencia de una imagen determinada para desarrollar cierto puesto de trabajo.

e) La difusión de estar en forma como sinónimo de delgadez.

\section{Definición de Percepción}

La percepción es un proceso mental activo mediante el cual la conciencia interpreta los estímulos sensoriales sobre objetos, hechos o situaciones a partir de los cuales se forman nuestras impresiones del mundo que nos rodea, los que van a ser influenciados por las experiencias, conocimientos, necesidades, intereses y emociones.

Escobar (2008) da a conocer que los componentes de la percepción son los siguientes:

\section{Componentes de la percepción}

a) Proceso de recepción sensorial: Para que se dé el proceso de la percepción es indispensableque hayan receptores sensoriales, ya sean visuales, auditivos, gustativos entre otros. Este proceso no está limitado a un solo campo sensorial, si no que se realiza simultáneamente.

b) Proceso de simbolización: La percepción está siempre asociada a un concepto, hay pues una aprehensión inmediata de un significado, de un estímulo intelectivo.

c) Proceso emocional: Muchas percepciones pueden dejarnos indiferentes, pero otras pueden estar estrechamente asociadas a un proceso emocional proporcionándonos placer, emoción, agrado o desagrado.

\section{Características de la percepción}

Escobar (2008) afirma lo siguiente sobre las principales características de la percepción:

a) Tiene una función adaptativa, en el sentido de que permite a la persona orientarse en su medio ambiente.

b) La percepción depende tanto de las características del objeto como de nosotros mismos.

c) Brinda estabilidad, en el sentido de que un objeto percibido es identificado aun cuando él o las circunstancias hayan variado en alguna medida. Tiene relación con esta característica la selección que hacemos de los aspectos más importantes del estímulo, pues ello es lo que le da la permanencia al estímulo.

d) Permite la búsqueda del significado ya que algunas veces la percepción es inmediata, en otras oportunidades no lo es, en cuyo caso tratamos de encontrarle un sentido, especialmente cuando lo que acontece afecta nuestro bienestar.

e) La percepción es posible aun cuando el estímulo presente pocos datos.

f) Percibimos en términos de probabilidades porque la experiencia nos enseña que ciertos objetos o acontecimientos van unidos entre ellos con cierto grado de probabilidad.

g) Bajo ciertas circunstancias la percepción puede ser equívoca y las dos formas más conocidas de error en la percepción son las alucinaciones y las ilusiones. 


\section{Percepción e imagen corporal}

Desde la psicología, Raich (2000) menciona que hay autores que cifran en un $25 \%$ el porcentaje de importancia de la percepción del cuerpo en nuestra autoestima.

Desde la perspectiva de la sociología, Zenón Pereyra nos explica que la marca de la religión, el mito, las ideologías, las costumbres y fundamentalmente la marca de las clases sociales, son mediaciones que fundan el modo de pensar, el sentir y el usar los cuerpos. Para el mismo Zenón somos intérpretes e interpretados en un constante diálogo corporal (Escobar, 2008).

\section{USO DE REDES SOCIALES Y PERCEPCIÓN DE IMAGEN CORPORAL}

Es importante destacar que la teoría de los procesos de comparación social de Festinger (1954), propone que las personas tienen una pulsión auto evaluativa, presentando una tendencia a usar información sobre los demás, para poder evaluar sus propias opiniones y habilidades.

Andsager (2014) también infiere, que dado que las mujeres están expuestas en las redes sociales a diferentes tipos de imágenes y fotos también se incentivaría diferentes tipos de cuerpos de mujeres como aceptables.

Adicionalmente, como menciona Perloff (2014), las personas con baja autoestima y los que tienen rasgos perfeccionistas pueden estar especialmente influenciados por las imágenes vistas en los medios lo que influye en la percepción de la imagen corporal.

En un reciente estudio de la Universidad de California realizado por Sherman \& otros (2015), se investigó la influencia de los pares de adolescentes, la medición de la conducta de los adolescentes y monitorización de las respuestas neuronales cuando se usan o reciben "me gusta" en las fotos. Se encontró que los adolescentes estaban más dispuestos a otorgar "me gusta" cuando las fotos tenían mayor cantidad de "me gusta" que las que tenían menores cantidades. La observación de fotos con mayores cantidades de "me gusta" está asociado a mayor actividad de áreas neuronales que implican el proceso de imitación, atención, procesos de reforzamiento y cognición social. Adicionalmente, se encontró que cuando los adolescentes miraban sus propias fotos con varios "me gusta", se activaron los mismos circuitos neuronales que cuando se come chocolate o se gana dinero.

Se podría inferir entonces, que existe influencia de las redes sociales, especialmente las que usan fotografías como principal medio de comunicación, en el comportamiento de las personas.

Un estudio realizado por Tiggemann \& Slater (2013) tuvo como objetivo principal examinar la relación entre la exposición a internet y la imagen corporal en adolescentes. Se encontró que el tiempo empleado en internet estaba significativamente relacionado a la internalización del ideal de delgadez, preocupación por el cuerpo y ansiedad por ser delgado. El 75\% de las encuestadas tenían un perfil de Facebook $\mathrm{y}$ fueron las que tuvieron un mayor puntaje en todos los ítems relacionados a la imagen corporal que las personas sin un perfil de Facebook.

Ellison, Steinfield \& Lampe (2007) también han examinado la relación entre el uso de las redes sociales y la salud psicológica de las personas. Dicho estudio encontró que los jóvenes con baja autoestima podrían beneficiarse del uso de la red social, ya que esto los ayuda a entablar relaciones interpersonales y mantener un lazo con otros, que de otra manera no harían.

Asimismo, Shaw \& Gant (2002) estudiaron algunos beneficios que puede traer consigo el "chatear" con otras personas, cómo esto puede afectar a las actividades sociales relacionadas a la autoestima, depresión, apoyo social y soledad. La investigación encontró que al "chatear" con otras personas que el usuario conoce poco o no conoce, los sentimientos de soledad y depresión decrecieron significativamente y la percepción de ayuda social y la autoestima incrementaron de manera significativa.

En tal sentido, Simoncic \& otros (2014) entrevistaron a 237 adultos jóvenes con el fin de examinar si el uso de Facebook estaba relacionado con síntomas depresivos y encontraron que no existe una relación directa entre el uso de Facebook y síntomas depresivos y, lo más resaltante fue concluir que, por 
el contrario, Facebook reducía síntomas de depresión en jóvenes mujeres que sufrían de altos niveles de características neuróticas, las que pueden ser un factor de riesgo para los desórdenes alimenticios.

Por tal motivo Simoncic \& otros concluyen que algunos tipos de interacción en redes sociales pueden tener un efecto positivo en la salud mental de las personas.

Adicionalmente, Cervera \& otros (2002), encontraron en un estudio con una muestra representativa de mujeres en España, que los altos niveles de síntomas neuróticos eran un mejor predictor de desórdenes alimenticios que una baja autoestima.

Ljepava \& otros (2013) encontraron que los usuarios frecuentes de Facebook tenían puntuaciones más altas relacionadas al narcicismo que los que no eran usuarios de dicha red social. Asimismo, Balakrishnan \& Shamim (2013) realizaron un focus group en Malasia y encontraron evidencias que sugieren que el uso frecuente de la red social podría estar relacionado a síntomas asociados a la adicción.

En Suecia se ha realizado un innovador tratamiento como primera etapa para combatir la bulimia nerviosa. Se trata de una combinación entre terapia conductual y un soporte social online a través del envío de correos electrónicos y discusiones en foros. Se pudieron detectar, además de las mejoras relacionadas a la percepción del cuerpo y la depresión, que algunas pacientes mejoraron también sus niveles de autoestima y satisfacción con su vida en general (Ljotsson \& otros, 2007).

Fichter \& otros (2012), analizaron la eficacia de llevar a cabo un programa basado en el uso de internet a manera de psicoterapia para tratar a mujeres jóvenes alemanas respecto a la prevención de recaídas de la anorexia nerviosa. La muestra fue de 258 jóvenes mujeres quienes fueron ubicadas de manera aleatoria en ambos grupos quienes se comprometieron por 9 meses a llevar un reporte y someterse a evaluaciones de especialistas clínicos quienes registraron la habilidad de evitar recaídas o, por el contrario, la falta de dichas habilidades.
El grupo que tenía la intervención mediante herramientas de internet, hacían uso de aplicaciones de interacción social incluyendo mensajes electrónicos que ofrecían apoyo de los demás, sesiones de chat y otras herramientas relacionadas.

Durante esos 9 meses, las mujeres pertenecientes al programa que usaba internet, ganaron más peso corporal, las ratios relacionados a las recaídas bajaron significativamente y lo más importante, los indicadores del tipo de psicopatología asociados a la anorexia nerviosa tuvieron cambios más favorables en dicho grupo.

Otro aspecto que aún falta ser estudiado relacionado a la imagen corporal, es el tamaño de los senos. En Estados Unidos y otras culturas occidentales, el ideal del cuerpo no está totalmente relacionado a ser delgado, sino a tener curvas (Harrison, 2003; Overstreet \& otros, 2010), lo cual se encuentra estrechamente relacionado con el acceder a cirugías plásticas.

Aunque se sabe muy poco sobre cómo el uso de las redes sociales puede influir en dicho aspecto, un estudio en Corea realizado por Davies \& Han (2011), muestra un vínculo entre cirugía cosmética y tecnología digital. Por ejemplo, hay celebridades que reciben cirugías gratuitas por promocionar a las clínicas que lo realizan en sus sitios web. También comunidades en línea y blogs se encuentran discutiendo y promocionando la cirugía cosmética lo que puede estar influenciando en los usuarios de las redes sociales.

\section{MÉTODO}

La presente investigación tiene una metodología cuantitativa y es de tipo no experimental. El diseño es de tipo transversal, descriptivo y correlacional ya que busca determinar la relación existente de dos variables: Uso de Facebook y Autopercepción de la Imagen Corporal.

La población es de 615 alumnas universitarias de la Facultad de Ciencias Sociales de una Universidad privada de Lima Metropolitana. La muestra es no probabilística y con un muestreo intencional, compuesto por 106 personas. 
Se utilizó la Escala de Intensidad de Facebook, el cuestionario incluye evaluaciones del comportamiento en Facebook y brinda información sobre en qué medida la encuestada participa activamente en las actividades de Facebook. Por otro lado, se utiliza una escala Likert, con opciones de respuesta que van desde totalmente de acuerdo a totalmente en desacuerdo, donde las participantes calificaron diversas afirmaciones. Esta escala ha sido usada en diversas investigaciones y ha mostrado un alto grado de confiabilidad $(\alpha=0.86)$ (Ellison, Steinfield \& Lampe, 2007). Así mismo, para la presente investigación, el cuestionario fue validado y arrojó un grado de confiabilidad de 0.824 .

Para medir la autopercepción de la imagen corporal se utilizó el Body Shape Questionnaire (BSQ). Este es un instrumento creado por Cooper, Taylor, Cooper y Fairbun en 1987 y adaptado a población limeña por Flores (2009). Su objetivo es evaluar el nivel de insatisfacción corporal considerando el miedo a engordar, los sentimientos de baja autoestima a causa de la apariencia y el deseo de perder peso. El BSQ está compuesto por 34 enunciados que miden la frecuencia con que aparecen ciertos pensamientos y emociones asociados con la imagen corporal. Los ítems son calificados en una escala likert de seis puntos: 1= nunca, $2=$ raramente, $3=$ a veces, $4=$ a menudo, $5=$ muy a menudo y $6=$ siempre. En el proceso de adaptación a la población Limeña, Flores (2009) contó con una muestra de 144 adolescentes femeninas, entre 16 a 21 años, pertenecientes a una universidad privada. Los resultados del análisis de confiabilidad arrojaron un alfa de Cronbach que demostró un alto nivel de consistencia al interior de la prueba $(\alpha=0.97)$. Así mismo, para la presente investigación, se obtuvo una confiabilidad de 0.97 . Para efectos del presente estudio se empleó el material adaptado en Lima previa autorización (Flores, 2009).

\section{RESULTADOS}

\section{Análisis descriptivo de las variables} Autopercepción de la Imagen Corporal.

Del total de encuestadas, el $56.6 \%$ se ha preocupado por su figura cuando ha estado aburrida, por lo menos a veces. Asimismo, el $65.2 \%$ por lo menos a veces se ha preocupado tanto por su figura que ha sentido que debería ponerse a dieta. El 34.9\% de encuestadas a menudo ha tenido miedo de engordar y el $32.1 \%$ se ha preocupado a menudo que su cuerpo no sea lo suficientemente firme. Finalmente, el $53.8 \%$ por lo menos a veces se ha sentido gorda al estar llena.

Del total de encuestadas, el $40.5 \%$ al estar rodeada de chicas delgadas, ha tomado conciencia de su propia figura, por lo menos a veces. Asimismo, el 37.7\% por lo menos a veces ha sentido que su figura se compara desfavorablemente al fijarse en la figura de otras chicas. El 17\% de encuestadas por lo menos a veces, pensar en su figura ha interferido en su capacidad de concentración. El 52.8\% de encuestadas por lo menos a veces, ha evitado ponerse ropa que la hace consciente de la forma de su cuerpo y el $63.3 \%$ se ha imaginado por lo menos a veces, reduciendo partes grasosas de su cuerpo.

Del total de encuestadas, por lo menos a veces el $36.8 \%$ se ha sentido gorda por comer comidas con muchas calorías. Asimismo, el $24.6 \%$ a veces se ha sentido avergonzada por su cuerpo. El $39.6 \%$ de las personas encuestadas asegura que por lo menos a veces preocuparse por su figura la ha hecho hacer dieta. El $24.6 \%$ afirma que a menudo ha pensado que la figura que tiene se debe a su falta de control. Finalmente, el $49.1 \%$ por lo menos a veces, se ha preocupado por que otras personas noten rollos en su cintura o estómago.

Del total de encuestadas, por lo menos a veces el $5.6 \%$ ha vomitado y el $8.5 \%$ ha tomado laxantes para sentirse más delgada. El $41.5 \%$ a veces se ha preocupado de que su cuerpo tenga aspecto de piel de naranja (celulitis) y el $38.7 \%$ de encuestadas afirma que a veces, ha evitado situaciones en las que las personas podrían ver su cuerpo. El 29.2\% por lo menos a veces se ha fijado más en su figura estando en compañía de otras personas. Finalmente, el $38.7 \%$ a menudo el preocuparse por su figura, le ha hecho sentir que debe hacer ejercicios.

\section{Uso de Facebook}

El $55.7 \%$ de las encuestadas tiene de 0 a 499 amigos en Facebook, el 27.4\% de las encuestadas tiene de 500 a 999 amigos en Facebook y el 16.9\% de las encuestadas tiene más de 1000 amigos en Facebook. 
El 21.7\% pasa más de 3 horas por día, haciendo uso de la red social Facebook.

El $25.5 \%$ por lo menos una vez al mes, actualiza su perfil de Facebook.

Dentro de las formas específicas de comunicación con amigos usando Facebook, el $80.2 \%$ de las encuestadas envía mensajes privados a un amigo. Así mismo, el 54.7\% usa el chat para comunicarse vía Facebook. El $45.3 \%$ envía mensajes grupales a sus amigos y el $44.3 \%$ Pone "like" en las fotos de sus amigos, actualizaciones de estado y publicaciones en muros. Finalmente, el $41.5 \%$ comenta en las fotos de sus amigos, actualizaciones de estado y publicaciones en muros.

El 55.7\% está de acuerdo con que Facebook es parte de su actividad diaria, el $30.2 \%$ se encuentra orgullosa de decir a las personas que tiene una cuenta de Facebook. Así mismo, el 30.2\% se siente fuera de contacto cuando no ha entrado a Facebook en un periodo de tiempo y el $57.5 \%$ está de acuerdo en que se siente parte de la comunidad de Facebook.

\section{Análisis de relación entre las variables \\ Relación entre las variables principales}

Se realizó un análisis de relación entre las variables objeto de estudio y se encontró una relación positiva y significativa entre el uso del Facebook y la autopercepción negativa de la imagen corporal $(\mathrm{r}=0.247 ; \mathrm{p}<0.05)$, lo cual significa que el uso de Facebook podría ser una variable importante en relación a la autopercepción de la imagen corporal.

\section{Relación entre las variables específicas}

Se encontró que el ciclo de estudios de las estudiantes parece no ser una variable importante en relación al uso de Facebook $\left(X^{2}=5.987 ; p>0.05\right)$.

Asimismo, se pudo comprobar que no existe una relación significativa entre la carrera de estudios y la autopercepción de la imagen corporal $\left(X^{2}=3.817\right.$; $\mathrm{p}>0.05)$.

De igual forma se encontró que el ciclo de estudios no sería una variable importante en relación a la autopercepción de la imagen corporal $\left(\mathrm{X}^{2}=7.741\right.$; $\mathrm{p}>0.05)$.
Finalmente se halló que no existe una relación significativa entre el ciclo de estudios y el tiempo diario de uso de Facebook $\left(X^{2}=8.518 ; p>0.05\right)$.

\section{DISCUSIÓN}

El propósito de la presente investigación fue corroborar la hipótesis que postula que existe una relación entre el uso de Facebook y la autopercepción de la imagen corporal en mujeres universitarias entre 17 y 24 años en una universidad privada de Lima Metropolitana.

Como se señala en los resultados, se pudo comprobar que el uso de la red social Facebook, influye en la autopercepción negativa de las mujeres, de su propio cuerpo.

$\mathrm{Si}$ bien es cierto, no se han encontrado investigaciones que estudien las mismas variables consideradas en el presente documento, se puede afirmar que diversos autores han llegado a conclusiones similares.

Por ejemplo, Rivarola (2003) realizó un estudio sobre la imagen corporal en adolescentes. En el grupo de 18 a 20 años se concluye que la insatisfacción con la propia imagen corporal estaría relacionada con los patrones socioculturales de la delgadez altamente valorados.

En ese sentido, es interesante observar cómo las redes sociales confluyen con dichos patrones socioculturales de la delgadez, afectando así el comportamiento de las personas vulnerables de manera casi inmediata.

Para corroborarlo, Chrisler \& otros (2013) realizaron un análisis de 977 tweets enviados antes y durante el desfile de modas de Victoria's Secret en 2011. Se pudieron detectar mensajes que contenían comparaciones con las modelos. Había tweets haciendo alusión a la imagen corporal, trastornos alimenticios, el peso, los deseos por la comida o el alcohol y pensamientos sobre autolesión. Los resultados apoyan la teoría de la comparación social y sugieren que los espectadores vulnerables podrían experimentar un efecto negativo o incluso participar 
en comportamientos nocivos, durante o después de ver dicho espectáculo u otros similares.

Se puede afirmar entonces que los resultados obtenidos en la presente investigación van de la mano con los resultados antes mencionados, ya que se ha podido comprobar el efecto que tiene en la autopercepción negativa de la imagen corporal, el hecho de que las mujeres estén más expuestas al uso de las redes sociales, especialmente Facebook y su contenido el cual alienta a la comparación del cuerpo, aspectos sociales, entre otros.

Es importante destacar en ese sentido, que la teoría de los procesos de comparación social de Festinger (1954) sigue vigente, ya que propone que las personas tienen una pulsión autoevaluativa, presentando una tendencia a usar información sobre los demás, para poder evaluar sus propias opiniones y habilidades.

Otro estudio realizado que concuerda con los resultados obtenidos en la presente investigación es el de Tiggemann \& Miller (2010) cuyo objetivo principal fue examinar la relación entre la imagen corporal y la exposición a diferentes medios de comunicación, especialmente Internet. Dicho estudio pudo determinar que la exposición a Internet y la lectura de las revistas, se encontraron relacionadas con una mayor internalización de ideales de delgadez, comparación de la apariencia física, insatisfacción con el peso y ansias por ser delgada.

Asimismo, en otro estudio que realizaron Tiggemann \& Slater (2013) que tuvo como objetivo principal examinar la relación entre la exposición a internet y la imagen corporal en adolescentes; encontraron que el tiempo empleado en internet estaba significativamente relacionado a la internalización del ideal de delgadez, preocupación por el cuerpo y ansiedad por ser delgado.

El $75 \%$ de las encuestadas tenían un perfil de Facebook y pasaban en promedio una hora y media diariamente en dicha red social. Cabe mencionar, que los usuarios de Facebook tuvieron un mayor puntaje en todos los ítems relacionados a la imagen corporal que las personas quienes no tenían un perfil de Facebook. Concluyeron que Internet representa un medio socio cultural muy relevante en la percepción de la imagen corporal en adolescentes mujeres (Tiggemann \& Slater, 2013).

Se puede concluir que los resultados obtenidos en la presente investigación han comprobado la relación inversa que existe entre el uso de Facebook y la satisfacción de la imagen corporal; la cual también es compartida por diversas investigaciones de manera indirecta, las cuales han sido mencionadas previamente.

\section{REFERENCIAS}

Acquisti,A.,\& Gross,R.(2006).Imagined communities: Awareness, information sharing, and privacy on the Facebook. In Privacy enhancing technologies, 36-58. Recuperado de http://people.cs.pitt.edu/ $\sim$ chang/265/proj10/zim/imaginedcom.pdf

Akyildiz, M., \& Argan, M. (2012). Uso de redes sociales online: los propósitos de estudiantes en el uso de Facebook en la Universidad de Turquía. Journal of Technology Research, 3, 111. Recuperado de: http://search.proquest.com/ docview $/ 1022983264$ ? accountid $=12268$

Andsager, J. L. (2014). Research Directions in Social Media and Body Image, Feminist Forum Commentary. doi: 10.1007/s11199-014-0430-4.

Baile, J. (2003). Imagen corporal. Centro Asociado de la UNED de Tudela, Recuperado de: http:// www.unedtudela.es/archivos_publicos/qweb_ paginas/4469/revista2articulo3.pdf

Balakrishnan, V., \& Shamim, A. (2013). Malaysian Facebookers: Motives and addictive behaviours unraveled. Computers in Human Behavior, 29, 1342-1349. doi:10.1016/j.chb.2013.01.010.

Bartholomew, M. K., Schoppe-Sullivan, S. J., Glassman, M., Kamp Dush, C. M., \& Sullivan, J. M. (2012). El uso de Facebook por parte de los nuevos padres en la transición hacia la paternidad. Family relations, 61(3), 455-469. Recuperado de http://www.ncbi.nlm.nih.gov/pmc/articles/ PMC3650729/

Boyd, D. M. \& Ellison, N. B. (2007). Social network sites: Definition, history, and scholarship. Journal 
of Computer Mediated Communication, 13(1), 210-230. Recuperado de: http://onlinelibrary.wiley. com/doi/10.1111/j.1083-6101.2007.00393.x/pdf

Cárdenas, C. (2015). La comunicación digital mediante redes sociales de organizaciones públicas. (Tesis inédita de Título Profesional). Universidad Nacional Mayor de San Marcos, Lima, Perú.

Cervera, S., Lahortiga, F., Martinez-Gonzalez, M. A., Gual, P., de Irala- Estevez, J., \& Alonso, Y. (2002). Neuroticism and low self-esteem as risk factors for incident eating disorders in a prospective cohort study. International Journal of Eating Disorders, 33, 271-280. doi: 10.1002/eat.10147.

Chrisler, J. C., Fung, K. T., Lopez, A. M., \& Gorman, J. A. (2013). Suffering by comparison: Twitter users' reactions to the Victoria's secret fashion show. Body Image, 10, 648-652. doi: 10.1016/ j.bodyim.2013.05.001.

Costa, J. (2009). El DirCom hoy: Dirección y Gestión de la Comunicación en la nueva economía. Barcelona: Costa Punto Com Editor.

Davies, G., \& Han, G.-S. (2011). Korean cosmetic surgery and digital publicity: Beauty by Korean design. Media International Australia, 141, 146156.

December, J. (1997). Notes on defining of computermediated communication. Computer-Mediated Communication Magazine, 3(1). Recuperado de: http://www.december.com/cmc/mag/1997/jan/ december.html

Delaney, J. (2013). El uso de Facebook y redes sociales por parte de los empleadores para discriminar a los postulantes a un empleo: Un análisis que sopesa los riesgos de tener una cuenta de Facebook y la necesidad de una legislación protectiva. Labor Law Journal, 64, 86-102. Recuperado de: http://search.proquest.com/ docview/1406216018? accountid=12268

DiVall, M. V., y Kirwin, J. L. (2012). Uso de Facebook para facilitar las conversaciones relacionadas a materias entre estudiantes y otros miembros de la Facultad. American journal of pharmaceutical education, 76(2). Recuperando de: http://iris.lib. neu.edu/cgi/viewcontent.cgi article $=1042 \&$ conte $\mathrm{xt}=$ bouve_fac_pubs
Duggan, M., Smith, A. (2014). Social media update 2013. Pew Research Center. Recuperado de http:// www.pewinternet.org/2013/12/30/social-mediaupdate-2013/

Ellison, N. B., Steinfield, C. \& Lampe, C. (2007). The benefits of Facebook "friends": Social capital and college students' use of online social network sites. Journal of Computer-Mediated Communication, 12, 1143-1168. doi:10.1111/ j.1083-6101.2007.00367.x.

Escobar, E. (2008). Percepción de la paciente mastectomizada sobre su imagen corporal en el Instituto Nacional de Enfermedades Neoplásicas. (Tesis inédita de Título Profesional). Universidad Nacional Mayor de San Marcos, Lima, Perú.

Festinger, L. (1954). A theory of social comparison processes. Human relations (2): 117-140.

Fichter, M. M., Quadflieg, N., Nisslmuller, K., Lindner, S., Osen, B., Huber, T., \& Wnsch-Leiteritz, W. (2012). Does internet-based prevention reduce the risk of relapse for anorexia nervosa? Behaviour Research and Therapy, 50, 180-190. doi:10.1016/ j. brat.2011.12.003.

Flores, M (2009). Validez y confiabilidad del body shape questionnaire en adolescentes universitarias de Lima Metropolitana. Tesis de Licenciatura en Psicología Clínica, Pontificia Universidad Católica del Perú.

Harrison, K. (2003). Television viewers' ideal body proportions: The case of the curvaceously thin woman. Sex Roles, 48, 255-264. doi:10. 1023/ A:1022825421647.

Hebrang Grgic, I. (2013). Bibliotecas escolares de Croacia en Facebook: encontrando las necesidades de jóvenes usuarios de bibliotecas. New library world, 114(9/10), 416-427. Recuperado de: http:// www.emeraldinsight.com/doi/abs/10.1108/NLW01-2013-0010

Internet World Stats (2016), Recuperado de: http:// www.internetworldstats.com

Ljepava, N., Orr, R. R., Locke, S., \& Ross, C. (2013). Personality and social characteristics of Facebook non-users and frequent users. Computers in Human Behaviors, 29, 1602-1607. doi:10.1016/j. chb.2013.01.026. 
Ljotsson, B., Lundin, C., Mitsell, K., Carlbring, P., Ramklint, M., \& Ghaderi, A. (2007). Remote treatment of bulimia nervosa and binge eating disorder: A randomized trial of internet-assisted cognitive behavioural therapy. Behavior Research and Therapy, 45, 649-661. doi: 10.1016/ j.brat.2006.06.010.

Morduchowicz,R.(2010). Los adolescentes y las redes sociales. Ministerio de Educación, Presidencia de la Nación, Recuperado de: https://issuu.com/ gcucuzza/docs/redes

Orellana, L. (2012). Uso de internet por jóvenes universitarios de la Facultad de Ciencias Sociales de la Universidad Nacional Federico Villarreal. (Tesis inédita de Maestría). Universidad Nacional Mayor de San Marcos, Lima, Perú.

Overstreet, N. M., Quinn, D. M., \& Agocha, V. B. (2010). Beyond thinness: The influence of a curvaceous body ideal on body dissatisfaction in black and white women. Sex Roles, 63, 91-103. doi:10. 1007/s11199-010-9792-4.

Palmer, A., \& Koenig-Lewis, N. (2009). Un enfoque experimental y basado en redes sociales hacia el marketing directo. Direct Marketing: An International Journal, 3(3), 162-176. Recuperado de: http://dx.doi.org/10.1108/17505930910985116

Park, H., \& Cho, H. (2012). Comunidades online de redes sociales: Fuentes de información para compra de vestimenta. The Journal of Consumer Marketing, 29(6), 400-411. Recuperado de http:// dx.doi.org/10.1108/07363761211259214

Perloff, R. (2014). Social Media Effects on Young Women's Body Image Concerns: Theoretical Perspectives and an Agenda for Research. Recuperado de School of Communication, Cleveland State University, 2121 Euclid Ave, Cleveland, OH 44115, USA. doi: 10.1007/s11199014-0384-6

Quiles, Y. (2003). Taller de Imagen corporal y alimentación, Recuperado de http://cpa.umh. es/files/2008/04/capitulo-4-taller-de-imagencorporal-y-alimentacion.pdf

Fecha de recepción: 10 de octubre, 2017

Fecha de aceptación: 5 de noviembre, 2017
Raich, M.(2000). Imagen Corporal: Conocer y valorar el propio cuerpo. Pirámide: Madrid.

Ressler, P., \& Glazer, G. (2010). Legislative: Nursing's engagement in health policy and healthcare through social media. OJIN: The Online Journal of Issues in Nursing, 16(1). Recuperado de: http:// www.nursing world.org/MainMenuCategories/ ANAMarketplace/ANAPeriodicals/OJIN/ Columns/Legislative/Health-Policy-andHealthcare-Through-Social-Media.html

Rivarola, M. (2003). La imagen corporal en adolescentes mujeres: su valor predictivo en trastornos alimentarios. Argentina.

Shaw, L. H., \& Gant, L. M. (2002). In defense of the internet: The relationship between internet communication and depression, loneliness, self-esteem and perceived social support. CyberPsychology \& Behavior, 5, 157-171. doi:10.1089/ 109493102753770552.

Sherman, L. E., Payton, A. A., Hernandez, L. M., Greenfield, P.M. \& Dapretto, M. (2015). The Power of the Like in Adolescence Effects of Peer Influence on Neural and Behavioral Responses to Social Media. Revista: Psychological Science. Recuperado de: http://pss.sagepub.com/content/ early/2016/05/24/0956797616645673.abstract

Simoncic, T. E., Kuhlman, K. R., Vargas, I., Hochins, S., \& Lopez-Duran, N. (2014). Facebook use and depressive symptomatology: Investigating the role of neuroticism and extraversión in youth. Computers in Human Behaviors, 40, 1-5. doi:10.1016/j.chb.2014.07.039.

Tiggemann, M., \& Miller, J. (2010). The internet and adolescent girls weight satisfaction and drive for thinness. Sex Roles, 63, 79-90. doi: 10.1007/ s11199-010-9789-z.

Tiggemann, M., \& Slater, A. (2013). NetGirls: The internet, facebook, and body image concern in adolescent girls. International Journal of Eating Disorders, 46, 630-633. doi:10.1002/eat.22141. 